\title{
SCIENTIFIC REPORTS

\section{OPEN Nondestructive real-space imaging of energy dissipation distributions in randomly networked conductive nanomaterials}

Received: 11 March 2019
Accepted: 19 September 2019 Published online: 10 October 2019

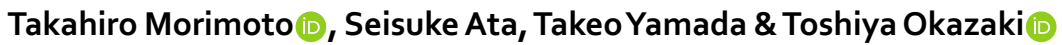

For realization the new functional materials and devices by conductive nanomaterials, how to control and realize the optimum network structures are import point for fundamental, applied and industrial science. In this manuscript, the nondestructive real-space imaging technique has been studied with the lock-in thermal scope via Joule heating caused by ac bias conditions. By this dynamical method, a few micrometer scale energy dissipations originating from local current density and resistance distributions are visualized in a few tens of minutes due to the frequency-space separation and the strong temperature damping of conductive heat components. Moreover, in the tensile test, the sample broken points were completely corresponding to the intensity images of lock-in thermography. These results indicated that the lock-in thermography is a powerful tool for inspecting the intrinsic network structures, which are difficult to observe by conventional imaging methods.
\end{abstract}

In the last few decades the progress of analysis and synthesis technologies has led to the discovery of truly various nanomaterials, such as carbon nanotubes $(\mathrm{CNTs})^{1}$, graphenes ${ }^{2}$, semiconducting nanowires ${ }^{3}$, transition metal dichalcogenides (TMDs) $)^{4,5}$ and metal nanoparticles ${ }^{6-8}$. These materials have unique properties originating from their geometrical structures, confined electronic structures and large specific surface areas. They are therefore attracting much attention as candidate materials for making new devices and materials having properties superior to those of conventional devices and materials, such as high-mobility devices ${ }^{9,10}$, flexible transparent devices ${ }^{11,12}$ and materials with high thermal and chemical resistances ${ }^{13}$. In many cases the nanomaterials consist of components randomly dispersed and oriented in two- or three-dimensional network structures called percolation states $^{14}$. For maximization of the various properties, how to control and analyze the geometric structures, surface conditions, junctions and space distributions are obviously important points if we are to realize the new functional devices and materials ${ }^{15,16}$. Especially in the case of electric and optical devices, visualization of the real current path is quite useful for understanding the intrinsic mechanism of the devices and materials.

For visualization of network structures and current distributions, some methods based on scanning probe microscopy (SPM) have been already reported ${ }^{17-25}$, e.g., scanning gate microscopy (SGM) as a moving gate technique $^{19}$ and conductive atomic force microscopy (C-AFM) as a moving electrode technique ${ }^{18}$. These SPM-based techniques have successfully visualized local current density and resistance distributions in nanometer-scale imaging. It is difficult, however, to image whole devices and amounts of materials large enough to determine the bulk properties because of the long time needed for image accumulation. Moreover, these methods are applicable only to two-dimensional devices or thin materials because their mechanisms are sensitive to surface conditions and sample thickness. Therefore, a faster and noncontact method is needed for more efficient investigation of bulk properties originating from nanomaterial network structures.

Here we propose a new method for multiscale systems that is based on the real-time lock-in thermography (LIT) technique ${ }^{26}$ with the Joule heating resulting from an ac bias conditions. The principal of this method was first suggested more than 40 years ago by Carlomagno ${ }^{27}$, and in the $80 \mathrm{~s}$ the camera based LIT technique was established in the nondestructive testing (NDT) of bulk materials ${ }^{28,29}$. For a long time, however, the limited resolution of infrared (IR) cameras restricted spread this useful method. In last decade the IR camera systems 

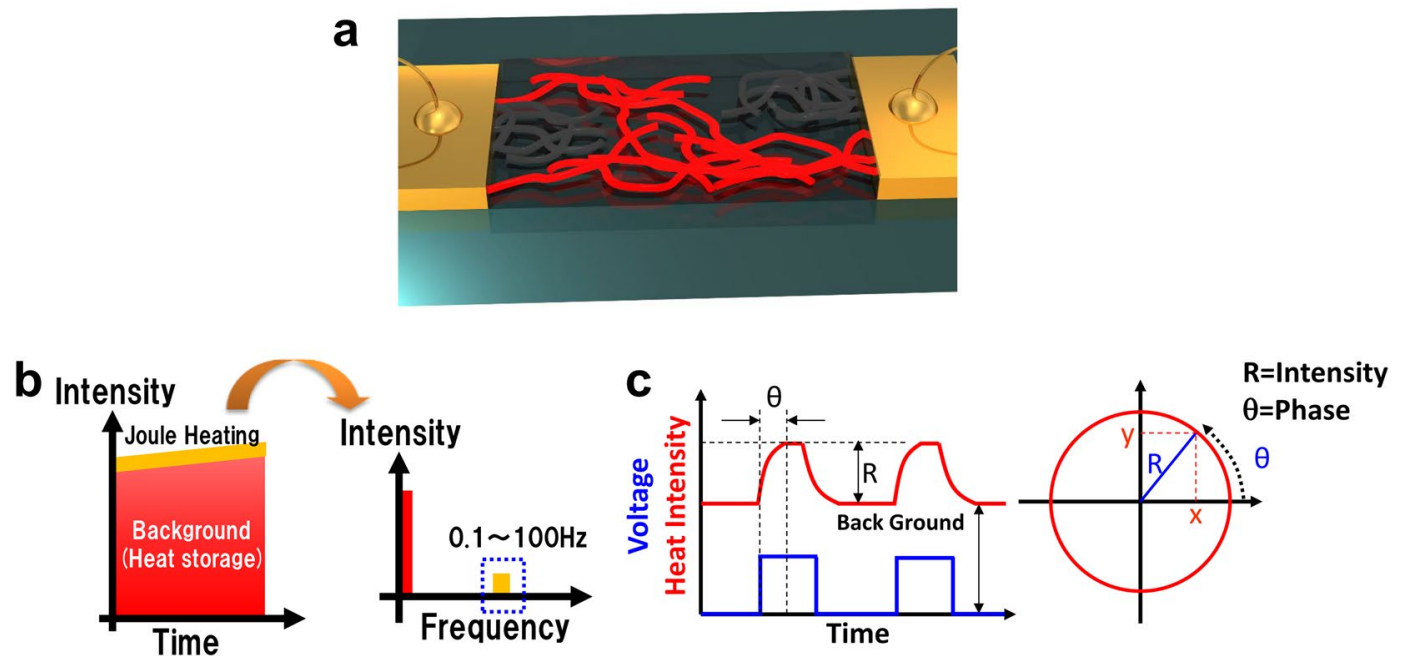

Figure 1. (a) Schematic illustration of experimental concept of the LIT method. The heat generated in conductive nanomaterial network structures is radiated from the surface. (b,c) Schematic image of the voltage and heat sequence and the LIT signal processing. In the ac bias voltage condition, the Joule-heating component has a frequency different from that of background heat storage. The applied bias voltage generates Joule heat with some delay time at rise and drop points. After the lock-in process, the heat intensity and phase are represented as $\mathrm{R}$ and $\theta$ in a complex vector diagram.

were dramatically improved, yielding more than a few hundred pixels in thermal microscope systems. Therefore, recently, in the field of failure analysis (FA) for integrated circuits, LIT is are gradually spreading as a fast testing method with a resolution higher than that of conventional laser scanning based methods ${ }^{30}$. The LIT technique is mainly used to improve sensitivity in FA and NDT because its signal-to-noise ratio $(\mathrm{S} / \mathrm{N})$ is higher than those of conventional plus or differential based methods ${ }^{31}$. In the nanomaterial research field, some manuscripts using a non-lock-in thermal microscope reported high-field breakdown in imaging with a heating condition ${ }^{32,33}$. In this study, however, we propose using this method to separate the wavelengths of various heat components originating from nanomaterial network structures in devices and materials (see details in the Supporting Information section S1). How to measure the heat component from only the nanomaterial networks is important for visualizing the energy dissipation depending on the current and resistance distributions in these randomly oriented systems.

\section{Results and Discussion}

Figure la shows the schematic concept of the LIT method. If we apply a bias voltage to conductive samples, the heat power density $(P)$ radiated should be given by a simple equation using the surface emissivity $(\varepsilon)$, Stefan-Boltzmann constant $(\sigma)$ and local temperature $(T)\left(P=\varepsilon \cdot \sigma \cdot T^{4}\right)$. In this case, the heat power should be rapidly spread to and stored in base materials. Therefore, the time-dependent temperature components can be given by

$$
T_{\text {Total }}=T_{\text {Joule }}(\theta)+T_{B G}(\text { const })+T_{\text {Diffuse }}(\theta+\Delta)
$$

where $\theta$ is the phase of the ac bias voltage and $\Delta$ is the delay time caused by the thermal conduction. Hence, the first term represents the Joule heating having same frequency as the applied bias voltage, the second term is the almost constant heat component corresponding to the background heat storage, and the third term is the thermal diffusive component of the non-active region near the heat source. The information of nanostructures might be contained in the first and third terms. In the case of dc bias voltage measurement, all heat component should be rapidly relaxed to constant background heat components, making it is impossible to measure the nanomaterial structures and local energy dissipation depending on the current density and resistance distributions. In the microscopic picture, the Joule heating originates from the electron-phonon interactions through the scattering phenomena. Therefore, the macroscopic Joule-heating signals are ensembles of scattering energy loss. For this reason, the intensity images obtained with the LIT method reflect the local power dissipation depending on the local voltage, resistance and current density in each position. The schematic images in Fig. 1b,c show the basic analysis process of the LIT method. By this method, the small heat components from the nanomaterials $(f=0.1-100 \mathrm{~Hz})$ are separated from the background component $(f=$ constant $)$ as shown in Fig. 1b. At this time, we use the ac bias frequency $25 \mathrm{~Hz}$, which is maximum value for full-pixel-size measurement in our system. In the real-time LIT method, the measured thermal frame images are calculated under measurement and then the intensity images $(\mathrm{R})$ and phase images $(\theta)$ are reconstructed. These processes are similar to those in the conventional dual-phase lock-in measurement in optical or electrical measurements.

Figure 2 a shows the optical microscope image of the tested sample. In this work we used as typical sample a conductive composite material that had relatively stable structures made of CNTs and polycarbonate (PC) base materials. Figure $2 b-e$ show IR and thermal images of the same sample shown in Fig. 2a. In infrared images obtained in non-bias conditions, the IR intensity is almost equal to the surface emissivity corresponding to the 


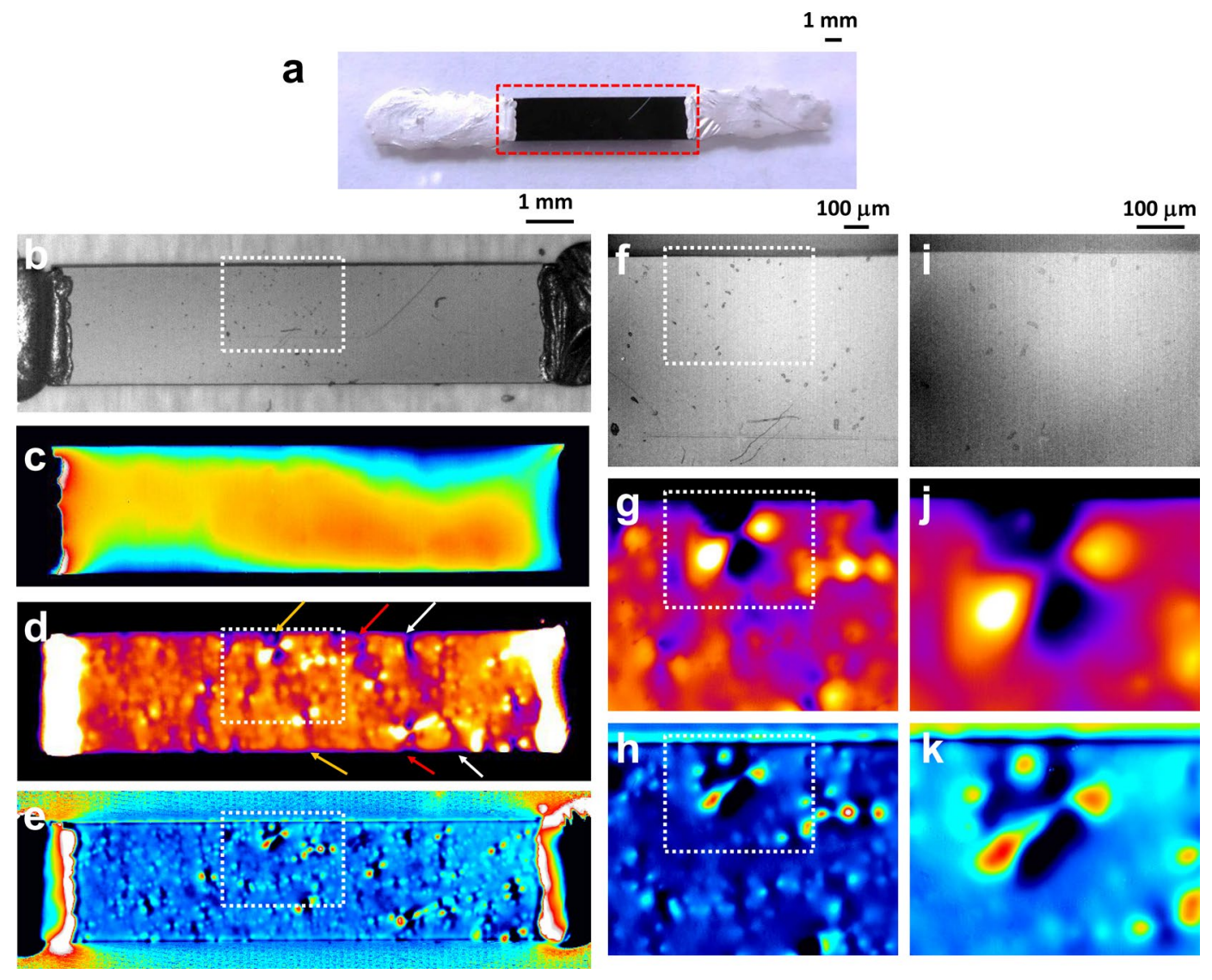

Figure 2. (a) Optical image of CNT/PC sample. Composite materials containing CNTs have an almost completely black surface, and the conventional optical method is not effective with these kinds of materials. (b-e) from top to bottom, low-magnification infrared, conventional-accumulation, intensity and phase images of the same sample shown in (a). In the infrared image, the sample showed a relatively flat and clean surface except for some particles and scratches. Moreover, the conventional method images showed only some blurred intensity distribution as shown in (c). The intensity (d) and phase (e) images show the heat distributions reflecting the network structures and local resistance. The pairs of arrow indicated in (d) indicate the higher-resistance cross sections across the current flow direction. (f-k) The middle- (f-h) and high- (i-k) magnification images show the pair of hot spots near the high-resistance cross section shown in (d). The narrow conductive channel and voltage concentration are clearly observed in these results. All LIT images were obtained by applying $60-\mathrm{V}$ and $6.4-\mathrm{mA}$ bias conditions and using 10 -minute accumulation.

surface concentration distribution of nanomaterials and base materials. Therefore, the relatively uniform surface seen in the IR image means that CNTs distributions are relatively uniform at the surface of this sample. Figure $2 \mathrm{c}$ shows the thermal image obtained with the conventional accumulation method under the same ac bias voltages. In this image the detailed structures are completely smeared out by the background heat storage to the base materials. On the other hand, the LIT phase and intensity images were quite different, as shown in Fig. 2d,e. Some high-resistance cross-section lines are clearly observed as the low-intensity signals indicated by same-color arrow-pairs in Fig. 2d. These regions may be corresponding to weakly linked network structures originating from the CNT dispersion or junctions. Figure $2 \mathrm{f}-\mathrm{h}$ show higher-magnification images of areas around the higher-resistance regions. In these images, the clear hot spot pair structures are observed near the high-resistance cross-section lines. In the central region of these hot spots, the quite narrow current path linking hot spots is also observed as shown in Fig. 2i-k. These structures indicate that the hot spot pair structures originate from the voltage concentration near the bottleneck structures of CNT networks. These results clearly show that the LIT method based on Joule heating detection can be used to visualize the internal current path and resistance distributions in complicated network structures consisting of randomly connected nanomaterials. Although the LIT method has relatively lower resolutions (a few micrometer) than the SPM based method (a few nanometer), this method has some advantages over the conventional method based on SPM systems. The required measurement time is quite short, ranging from a few tens of seconds to a few tens of minutes, and is typically less than ten minutes when there is enough heat radiation and DC power $(\sim \mathrm{mW})$. The field of view of one measurement is also very large-scale more than $1.0 \mathrm{~cm}$ in the lowest magnification. This scalability is important to investigate the mechanism of bulk properties based on the microscopic phenomena. Moreover, the images were obtained under completely nondestructive conditions without heating damage. This is obviously important for reproducibility and additional measurement with other methods at the same position and under the same conditions. 


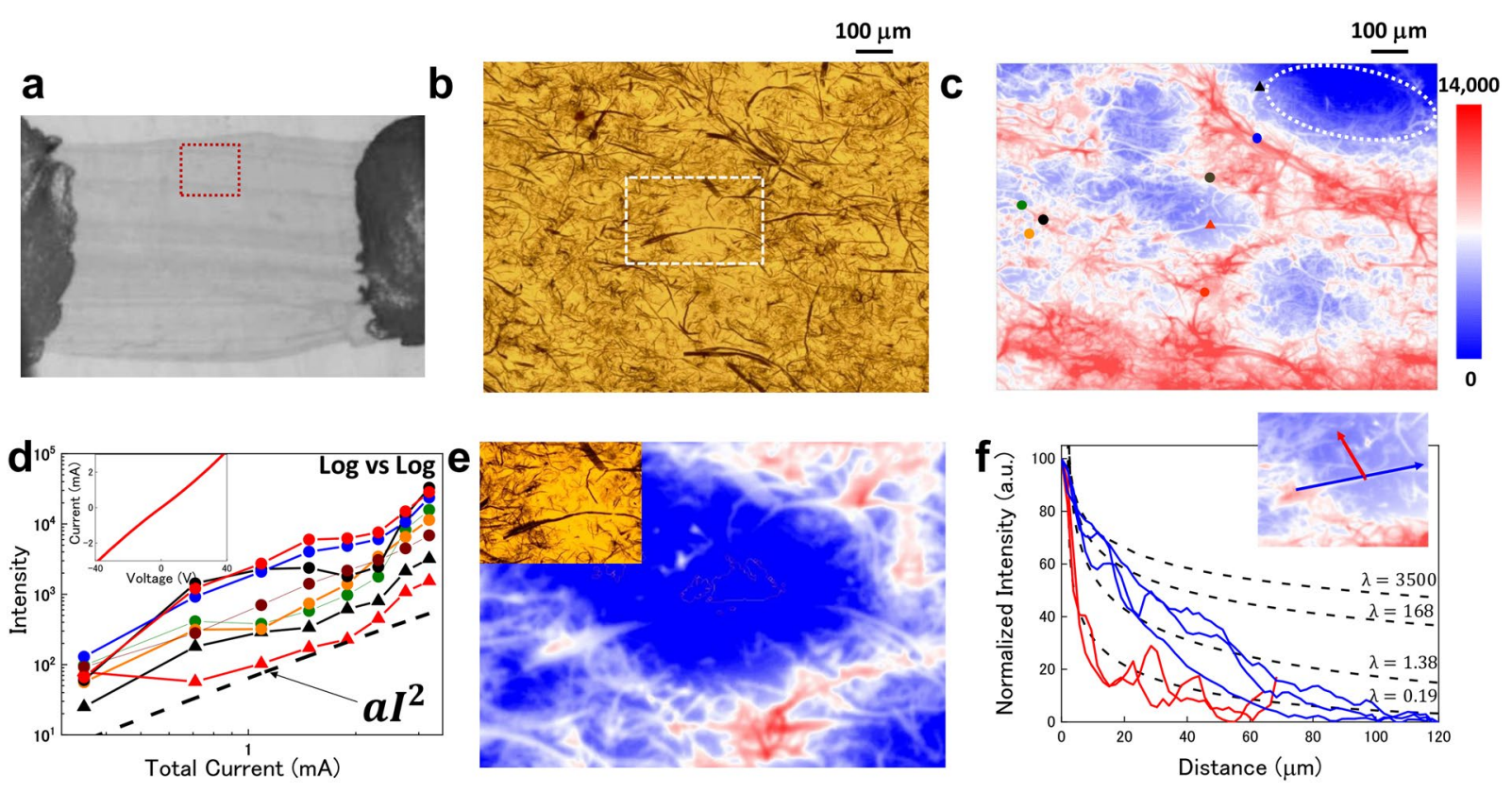

Figure 3. (a) Infrared image of the measured sample. The red-dotted square shows the measured area in $(\mathbf{b}, \mathbf{c})$. (b) Transmission optical microscope image at same area as c. The area in the white-dotted box is same area as shown in the inset of (e). (c) The LIT intensity image obtained with a high-magnification IR lens ( $\mathrm{x} 8$, N.A. $=0.75)$. The image area is $1200 \mu \mathrm{m} \times 960 \mu \mathrm{m}$. Each symbol corresponds to a measured point shown in (d). The dashed white circle indicates the LIT non-active region. (d) The log-scale intensity as function of the log-scale total current. All the curves show the clear Joule-heating dependence of the total current. Here a is a pre-factor. Inset: The I-V characteristic of the same sample. (e) The phase-separation image $\left(I_{L I T}=R \cos \theta\right)$ for the in-phase component of ac bias voltage with same color range as shown in (c). The signal from the heat source disappeared in the heat-conductive region. The optical microscope image of the white-dotted area in (b) is shown in the inset. (f) The comparison between theoretical estimation curves and LIT intensity. The red curves represent the thermal conduction to the FKM. The blue curves represent the thermal conduction to CNTs. Dashed lines corresponding to the thermal conductivity of FKM (0.19), quartz (1.38), silicon (168) and a single CNT (3500). These regions are chosen from the non-heat source regions as shown in (e). One example of chosen point at the FKM and CNT is shown in the inset.

Although the LIT images are made using the sample surface radiations, they are the ensembles of the signals delivered from the different depth positions within the thermal diffusion length $(\Lambda)$, which is simply described as $\Lambda=\sqrt{2 \alpha / f}$, where $\alpha$ is the thermal diffusivity and $f$ is the ac bias frequency. In the case of $25 \mathrm{~Hz}$ measurement, the CNT composite materials show a $\Lambda$ greater than $2.0 \mathrm{~mm}$. This means that a thin sample should show clearer LIT images in higher-magnification imaging. Figure $3 a, b$ show the IR and optical microscope images of a sample less than $1 \mu \mathrm{m}$ thick that was made by using a cryo-microtome. In these images, it is difficult to estimate the current path in these structures. On the other hand, the LIT image clearly shows the distribution of current in the complicated network structures as shown in Fig. 3c. In the CNT network structure samples, the total resistance might be simply described as ensembles of the contact resistance $\left(R_{c}\right)$, CNT resistance $\left(R_{C N T}\right)$ and CNT junction resistance $\left(R_{j}\right)$, as described by

$$
\mathrm{R}(V, T)=R_{C}+R_{C N T}+R_{j}
$$

here, second term is described as $\left(R_{C N T}=h / 4 e^{2} \cdot\left(L+\lambda_{\text {eff }}\right) /\left(\lambda_{\text {eff }}\right)\right)$, where the effective mean free path (MFP) $\left(\lambda_{e f f}\right)$ is estimated from the acoustic phonon MFP $\left(\lambda_{A C}\right)$ and optical phonon MFP $\left(\lambda_{O P}\right)$ as $\lambda_{\text {eff }}=\left(\lambda_{A C}^{-1}+\lambda_{O P}^{-1}\right)^{-134}$. Third term is described as $\left(R_{j}=\alpha T_{1} /\left(T_{0}+T\right)\right)$, where $T_{0}$ and $T_{1}$ are Sheng's parameters corresponding to the tunneling transport ${ }^{35}$. In the self-heating condition under higher bias voltages, the $R_{C N T}$ and $R_{j}$ should show the non-linear characteristics via changing of the electron phonon scattering rate and the tunneling rate through the potential barriers in CNT junctions ${ }^{34,36}$. The I-V characteristic of this sample showed almost linear dependence as shown in inset of Fig. $3 \mathrm{~d}$. This means that the bias condition in this measurement is sufficiently small, and the energy dissipation is simply considered within the classical electronic circuit models. Therefore, the LIT image intensity should be enhanced in the lowest resistance region in parallel current path and highest resistance region in series current path. Figure $3 \mathrm{~d}$ shows the LIT intensity at various positions as functions of total current for the whole device. All signal intensities show clear dependence proportional to $I^{2}$ and this indicates that the signal should be originated from the only Joule heating by the LIT process. Another important point is the non-active region observed at the top-right of Fig. 3c. Although the CNTs exist in these regions, the LIT intensity signal is insignificantly small. This means that these CNTs should be electrically isolated from the current path and should not contribute to bulk properties. How to reduce the occurrence of non-active regions and realize uniform 
distributions is important for making bulk materials and devices with better properties. Therefore, the LIT technique should be useful for optimizing the microscopic structure and the sample fabrication process.

In thin-sample imaging, the resolution is dramatically better than bulk-sample results. The resolution of the LIT image of an isolated CNT is near the diffraction limit $(\sim 2.0 \mu \mathrm{m})$ of the wavelengths detected (from 3.0 to $5.0 \mu \mathrm{m})$. This resolution improvement is simply explained by strong damping of thermal conduction in ac bias conditions. In the case of a "thermally thin" sample (one with a thickness significantly smaller than the thermal diffusion length), the heat source should be considered as a mirror heat source chained by multiple reflections at the interfaces within the conduction path. Therefore, the temperature conduction is described as cylindrical thermal conduction from a line-like heat source by using Kelvin functions ${ }^{26}$.

$$
T(r, t)=A\left(\operatorname{ker}\left(\frac{r \sqrt{2}}{\Lambda}\right)+i k e i\left(\frac{r \sqrt{2}}{\Lambda}\right)\right) e^{i \omega t}
$$

Here $\Lambda$ is the thermal diffusion length as mentioned above, $\omega$ is the angular frequency $\left(f_{\text {lock-in }}=\omega / 2 \pi\right)$, and ker and $k e i$ are Kelvin functions of the second kind. The LIT intensity should therefore be proportional to $\sqrt{(k e r)^{2}+(k e i)^{2}}$. In the LIT, the intensity image could be separated in each phase component described as $I_{L I T}=R \cos \theta$ (see in detail in section S2). If we plot the faster in-phase component, only the Joule heating current path is imaged as shown in Fig. 3e. Actually, in this image, the signal of the broken CNT is disappeared, and this means that this CNT signal consisted only of the thermal conduction component from the heat source. Therefore, it is possible to verify the quantitative analysis of the thermal conduction in these regions. Figure $3 \mathrm{f}$ shows the comparison between the LIT intensity profile and theoretical curves estimated from bulk thermal parameters (see in detail in section S3). In the case of thermal conduction from a heat source to FKM, the strongly damped curve has good correlations to the theoretical curve. On the other hand, the longer-distance signals of the CNT bundle show slight differences from the fitting curve estimated from the thermal conductivities of CNTs ${ }^{34,37-39}$. The more strongly damped curve of CNTs might originated from the thermal diffusion to FKM. Although the real temperature value is subtracted in the LIT process, these results clearly indicate that the LIT method is also useful for estimation of thermal properties in microscopic structures.

In the LIT images, the heat intensity should be influenced by the local electric structures depending on the CNTs connectivity as shown in Fig. 2. Therefore, in our method the distributions of current paths can be quantitatively analyzed through the intensity distribution. In Fig. 4a-d the density-dependent results of CNT/FKM composite materials are shown for the same sample size and same applied DC power conditions $\left(P_{D C}=V_{D C} \times I_{D C}\right)$. The inhomogeneity of LIT images is gradually smeared out by formations of uniform network structures by increasing CNT concentrations. In the case of the $5.0 \%$ sample, the intensity fluctuation is quite a bit smaller than those of lower-density samples. Reflecting these uniform network structure and CNT density increment, the sample resistances are also dramatically decreased, and peak shapes have narrow and near normal distributions as shown in Fig. 4e,f. For more quantitative analysis, the standard deviation (SD) are estimated from the intensity distributions (see in detail section S4). Figure $4 \mathrm{~g}$ shows the SDs as a function of normalized resistance in samples with each CNT concentration. Although these normalized resistances still contained contact resistance, the SD has clear dependence on the $\log (R)$ originated from percolation behaviors in these systems ${ }^{15,16}$. On the other hand, in the lower CNT concentration region, the power law dependence of the CNT concentration vs LIT SDs showed the deviation from linear dependence as shown in the inset of Fig. 4g. This might be caused by the LIT-nonactive isolated small clusters as shown in Fig. $3 c^{40}$. Although it is difficult to estimate the percolation threshold by the LIT method, the quantitative evaluation of nanomaterial network structures is useful for process and property optimization in various materials and devices containing conductive nanomaterials.

If the LIT images are really reflecting the whole current distributions and network structures, these signals should be directly correlated with physical-mechanical properties like those evaluated in the tensile testing of bulk materials. Figure 5 shows the tensile test results and LIT intensity images obtained at the same time in the same positions. The test piece was formed dumbbell-shaped to reduce stress concentration at the edge of the sample as shown in Fig. 5a. Before tensile testing (Fig. 5b,c), the sample had two higher-thermal-intensity regions as shown in Fig. 5c,d. These strongly power-dissipated points mean that the local structure has bottlenecks of the current paths. These high-heating points consisted of weakly linked network structures corresponding to the existence of weakly linked CNT junctions. Therefore, these heating spots should be related to the physical-mechanical properties of bulk materials.

Figure 5e,f showed the IR and LIT images measured at 40\% strained conditions, respectively. Applying strain in the horizontal direction obviously extended the sample length in the strain direction. This tensile effect is also observed in the I-V characteristics as shown in Fig. 5h. The sample resistance clearly increased from $25.9 \mathrm{k} \Omega$ to $35.4 \mathrm{k} \Omega$ at $40 \%$ strain. In this step, the LIT images also indicated difference from the initial state on their intensity profiles. The intensities of hot spots in the initial state are more emphasized by increasing local resistance. Moreover, the top of sample center region already showed dramatically decreased LIT intensity caused by broken internal network structures of CNTs. This prediction is confirmed in the next step results at $45 \%$ strain. In this condition, the sample started to break at two different points as shown in Fig. 5g. Both of them correspond to the higher LIT intensity region in the initial state. This means that the LIT intensity distributions are clearly related to physical-mechanical properties through the power dissipation distributions reflecting network structures of conductive nanomaterials. These results show that the LIT method will enable measurements to be made by another method before destructive tensile testing.

In this manuscript, the basic concept and typical results of the LIT method were discussed based on its use in conductive composite materials. This method has also wide applicability for conductive materials and 


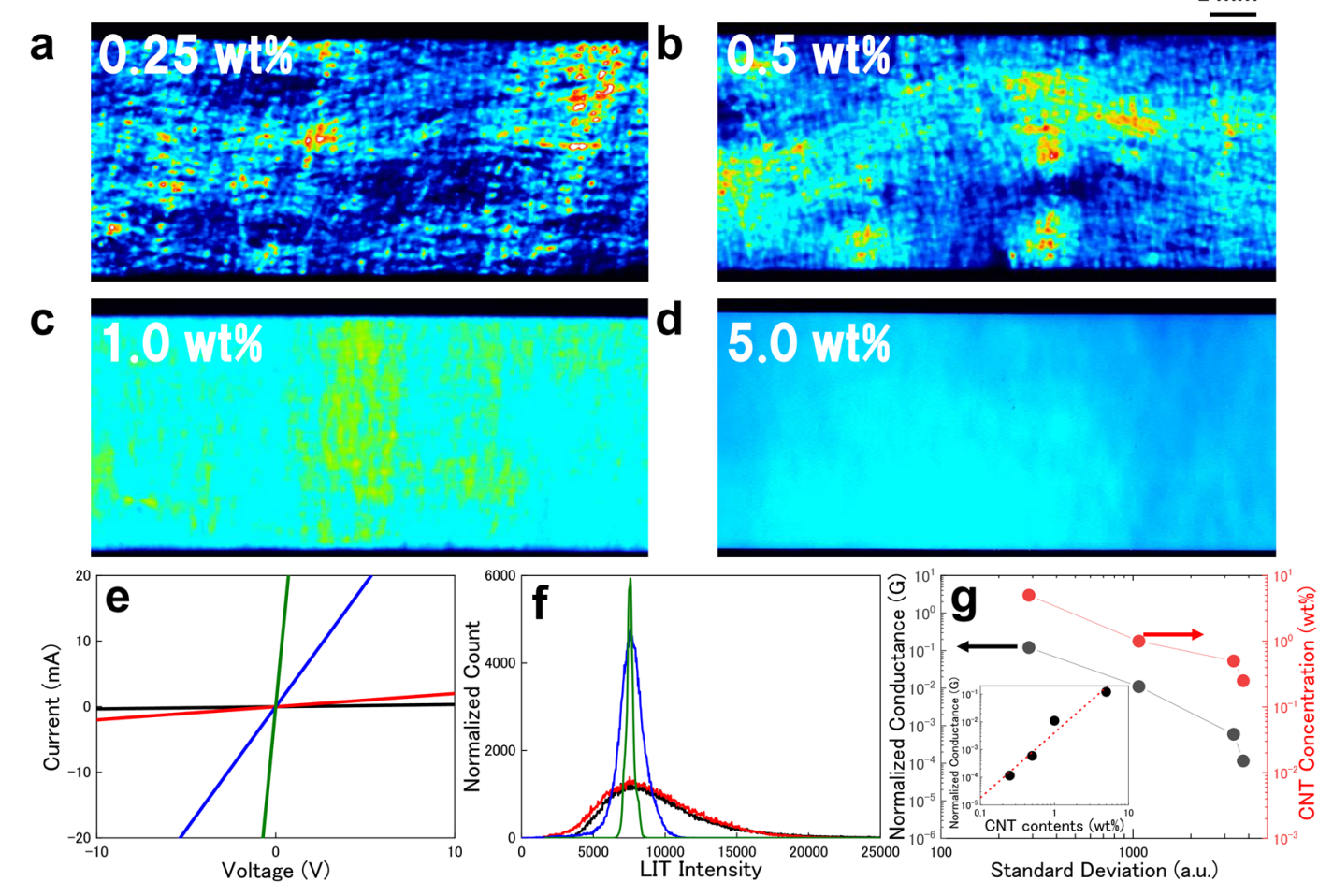

Figure 4. (a-d) Density-dependent LIT intensity images for CNT contents from $0.25 \mathrm{wt} \%$ to $5.0 \mathrm{wt} \%$. (e) The I-V characteristics of each sample. All data show clear linear dependence within the range of applied voltages. (f) Normalized count numbers shown as a function of the LIT intensity. The count numbers are normalized by the difference of sample size and image pixels. (g) Standard deviations of intensity distributions shown as a function of normalized sample resistance. The resistances are corrected by sample dimensions. Inset: The LIT standard deviation (left, black) and normalized conductance (right, red) shown as a function of the CNT concentrations in log-log scale plots.

devices. Therefore, we also report the results of its use on the low-dimensional graphene devices in a following manuscript ${ }^{41}$.

\section{Conclusion}

We have investigated the LIT method as a nondestructive real-space method for imaging the energy dissipation depending on the current density and local resistance, mechanical properties and space distributions for randomly networked nanomaterials. The small Joule heating of the nanomaterials was detected in frequency-space-separation images made using the lock-in thermography technique. These lock-in thermal images were directly correlated with energy dissipations and physical-mechanical properties reflecting inhomogeneity of conductive nanomaterial network structures. This is quite important for various investigations of the fundamental mechanisms related carrier dynamics inside nanomaterial devices and materials. Moreover, the image accumulation faster than that of conventional SPM-based methods is also important in applied physics and industrial science for multiscale measurements at scales from microscopic to macroscopic. Therefore, the lock-in thermography technique should be important tool for realization the more useful functional devices and materials with various nanomaterials.

\section{Methods}

The LIT measurement. The LIT measurement was performed by using a lock-in thermography system for failure analysis (Themos-1000, Hamamatsu). IR signals with wavelengths from 3.0 to $5.0 \mu \mathrm{m}$ were detected by the system's InSb camera. The electrical transport characteristics under an ac bias were evaluated by using a B1500 semiconductor parameter analyzer (Keysight). The electrodes were made from silver conductive pastes, and electrical probing was done using the home-made probe systems. The ac bias voltages were applied as rectangular signals having a $50 \%$ duty ratio. All the measurements were done at $25 \mathrm{~Hz}$, whereas the camera's maximum frequency was $100 \mathrm{~Hz}$. Therefore, four IR images were stored in each cycle of the lock-in process.

Sample fabrication. In the work reported in this manuscript, we used two different CNTs and two different base materials for composite samples. The super-growth and HiPCo single-walled CNTs were kneaded with a polycarbonate (PC, Panlight L-1225Y, Teijin Limited) and a fluoro-rubber (FKM, Daiel G912, Daikin Industries, Ltd.). The SG/PC samples were used in the experiment of Figs 1 and 2. The SG/FKM samples were used in the measurement of Figs 3 and 4. In the tensile test shown in Fig. 5, the HiPCo/FKM sample had strain behaviors suitable for the LIT measurement. The SG/PC samples were provided by a biaxial continuous 

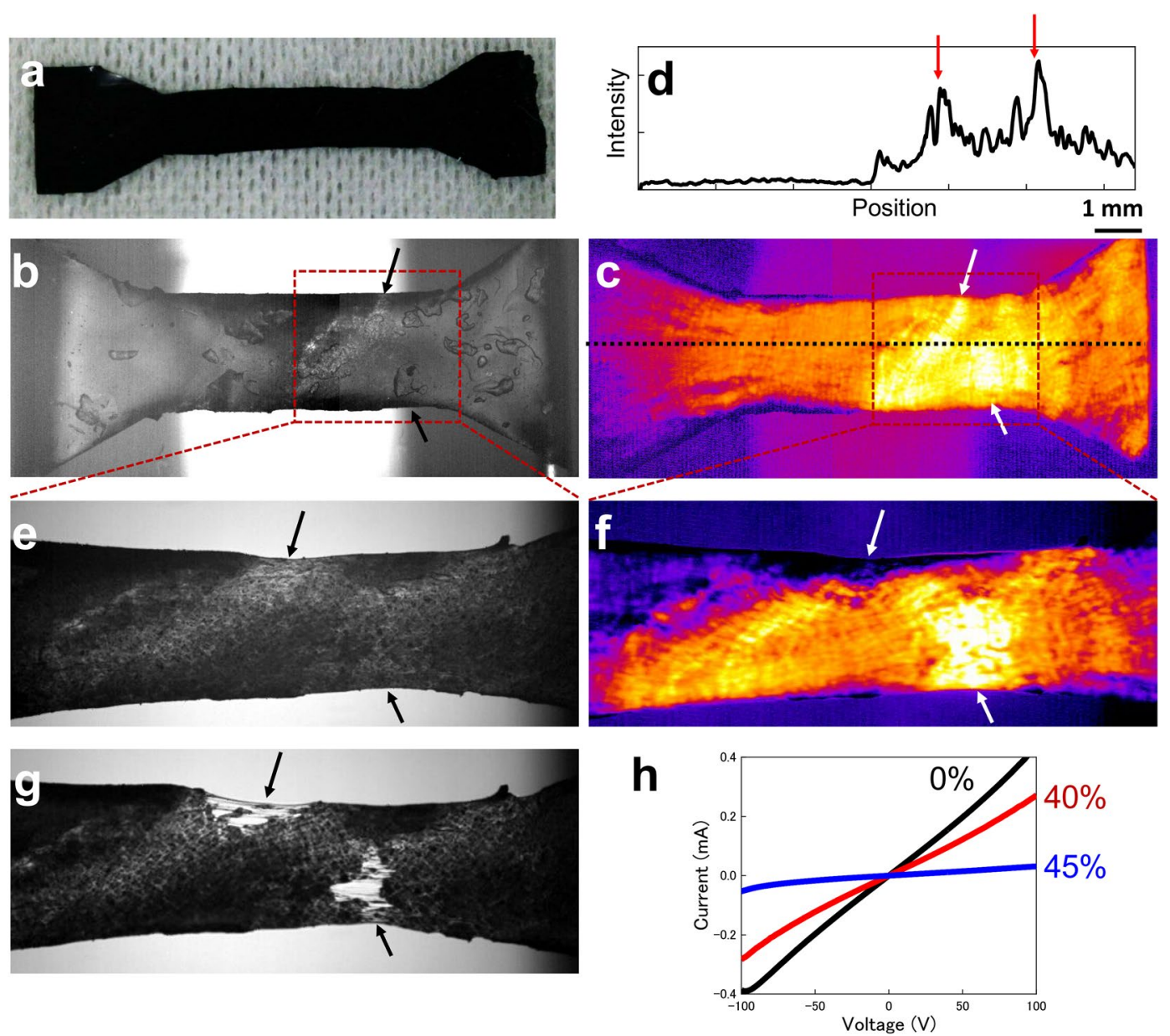

Figure 5. (a) Optical microscope image of sample made from same rubber sheet measured in tensile test. $(\mathbf{b}-\mathbf{g})$ The IR and LIT intensity images obtained during tensile testing at initial condition (b,c) and at $40 \%$ strain $(\mathbf{e}, \mathbf{f})$. (d) Cross section of intensity profile at initial state shown in (c) (dashed line). The arrows indicate the strong heating points corresponding to the high-resistance region. (g) The sample has broken at 50\% strain. All arrow corresponds to the high-intensity region at initial condition. (h) The I-V characteristics in each tensile condition. The two-terminal resistance was changed from $25.9 \mathrm{k} \Omega$ to $1.9 \mathrm{M} \Omega$.

kneader (Explore, MC-15TS, Explore Instruments) and formed with injection molding (Explore, 12cc, Explore Instruments). The SG/FKM and $\mathrm{HiPCo} / \mathrm{FKM}$ samples were fabricated by a drop casting method. Except in the concentration-dependence measurement, the CNT concentration was $1.0 \mathrm{wt} \%$ to the base materials (PC and FKM). The thin sample measured in Fig. 4 was made by using the RM2265 cryo-microtome (Reica) at liquid-nitrogen temperature.

\section{References}

1. Iijima, S. Helical microtubules of graphitic carbon. Nature 354, 56-58 (1991).

2. Novoselov, K. S. Electric Field Effect in Atomically Thin Carbon Films. Science 306, 666-669 (2004).

3. Holmes, J. D., Johnston, K. P., Doty, R. C. \& Korgel, B. A. Control of thickness and orientation of solution-grown silicon nanowires. Science 287, 1471-1473 (2000).

4. Mak, K. F., Lee, C., Hone, J., Shan, J. \& Heinz, T. F. Atomically thin MoS2: A new direct-gap semiconductor. Phys. Rev. Lett. 105, 2-5 (2010).

5. Splendiani, A. et al. Emerging photoluminescence in monolayer MoS2. Nano Lett. 10, 1271-1275 (2010).

6. Yanson, A. I., Rubio Bollinger, G., Van Den Brom, H. E., Agraï, N. \& Van Ruitenbeek, J. M. Formation and manipulation of a metallic wire of single gold atoms. Nature 395, 783-785 (1998).

7. Kondo, Y. \& Takayanagi, K. Synthesis and characterization of helical multi-shell gold nanowires. Science 289, 606-608 (2000).

8. Hong, B. H., Bae, S. C., Lee, C. W., Jeong, S. \& Kim, K. S. Ultrathin single-crystalline silver nanowire arrays formed in an ambient solution phase. Science 294, 348-351 (2001).

9. Lin, Y.-M. et al. Operation of Graphene Transistors at Gigahertz Frequencies. Nano Lett. 9, 422-426 (2009).

10. Kim, S. et al. Realization of a high mobility dual-gated graphene field-effect transistor with $\mathrm{Al}_{2} \mathrm{O}_{3}$ dielectric. Appl. Phys. Lett. 94, 062107 (2009).

11. Wu, Z. et al. Transparent, conductive carbon nanotube films. Science 305, 1273-1276 (2004).

12. Hu, L., Kim, H. S., Lee, J. Y., Peumans, P. \& Cui, Y. Scalable coating and properties of transparent, flexible, silver nanowire electrodes. ACS Nano 4, 2955-2963 (2010).

13. Ata, S., Tomonoh, S., Yamda, T. \& Hata, K. Improvement in thermal durability of fluorinated rubber by the addition of single-walled carbon nanotubes as a thermally stable radical scavenger. Polymer 119, 112-117 (2017). 
14. Broadbent, S. R. \& Hammersley, J. M. Percolation processes: I. Crystals and mazes. In Mathematical Proceedings of the Cambridge Philosophical Society 53, 629-641, (Cambridge University Press, 1957).

15. Bauhofer, W. \& Kovacs, J. Z. A review and analysis of electrical percolation in carbon nanotube polymer composites. Compos. Sci. Technol. 69, 1486-1498 (2009).

16. Mutiso, R. M., Sherrott, M. C., Rathmell, A. R., Wiley, B. J. \& Winey, K. I. Integrating simulations and experiments to predict sheet resistance and optical transmittance in nanowire films for transparent conductors. ACS Nano 7, 7654-7663 (2013).

17. Williams, C. C. \& Wickramasinghe, H. K. Scanning thermal profiler. Appl. Phys. Lett. 49, 1587-1589 (1986).

18. Murrell, M. P. et al. Spatially resolved electrical measurements of $\mathrm{SiO}_{2}$ gate oxides using atomic force microscopy. Appl. Phys. Lett. 62, 786-788 (1993).

19. Eriksson, M. A. et al. Cryogenic scanning probe characterization of semiconductor nanostructures. Appl. Phys. Lett. 69, 671-673 (1996).

20. Varesi, J. \& Majumdar, A. Scanning Joule expansion microscopy at nanometer scales. Appl. Phys. Lett. 72, 37-39 (1998).

21. Topinka, M. A. et al. Imaging coherent electron flow from a quantum point contact. Science 289, 2323-2326 (2000).

22. Topinka, M. A. et al. Coherent branched flow in a two-dimensional electron gas. Nature 410, 183-186 (2001).

23. Okigawa, Y., Kishimoto, S., Ohno, Y. \& Mizutani, T. Electrical properties of carbon nanotube thin-film transistors fabricated using plasma-enhanced chemical vapor deposition measured by scanning probe microscopy. Nanotechnology 22 (2011).

24. Jeong, H. \& Park, J.-Y. Local Electrical Investigations of Nitric Acid Treatment Effects on Carbon Nanotube Networks. J. Phys. Chem. C 119, 9665-9668 (2015).

25. Grosse, K. L. et al. Direct observation of resistive heating at graphene wrinkles and grain boundaries. Appl. Phys. Lett. 105, 143109 (2014).

26. Breitenstein, O. \& Langenkamp, M. Lock-in Thermography. 10, (Springer Berlin Heidelberg, 2003).

27. Carlomagno, G. M. \& Berardi, P. G. Unsteady Thermotopography in Non-Destructive Testing. In Proc. 3rd Biannual Exchange (IRIE '76) 2, 33-40, (McGraw-Hill Book Co, 1976).

28. Beaudoin, J. L. et al. Numerical System For Infrared Scanners And Application To The Subsurface Control Of Materials By Photothermal Radiometry. Infrared Technol. Appl., SPIE 0590, 285-292 (1986).

29. Kuo, P. K., Ahmed, T., Jin, H. \& Thomas, R. L. Phase-Locked Image Acquisition in Thermography. Proc. SPIE 41-47 (1988).

30. Nikawa, K., Inoue, S., Morimoto, K. \& Sone, S. Failure analysis case studies using the IR-OBIRCH (infrared optical beam induced resistance change) method. Test Symp. 1999. (ATS '99) Proceedings. Eighth Asian 394-399 (1999).

31. Meola, C., Carlomagno, G. M. \& Giorleo, L. The use of infrared thermography for materials characterization. J. Mater. Process. Technol. 155-156, 1132-1137 (2004).

32. Estrada, D. \& Pop, E. Imaging dissipation and hot spots in carbon nanotube network transistors. Appl. Phys. Lett. 98, 96-99 (2011).

33. Gupta, M. P. et al. High field breakdown characteristics of carbon nanotube thin film transistors. Nanotechnology 24 (2013).

34. Pop, E. et al. Negative differential conductance and hot phonons in suspended nanotube molecular wires. Phys. Rev. Lett. 95, 1-4 (2005).

35. Ping, S. Fluctuation-induced tunneling conduction in disordered materials. Physical Review B 21 (1980).

36. Fuhrer, M. S. et al. Crossed Nanotube Junctions. Science 288, 494-497 (2000).

37. Pop, E., Mann, D., Wang, Q., Goodson, K. \& Dai, H. Thermal conductance of an individual single-wall carbon nanotube above room temperature. Nano Lett. 6, 96-100 (2006).

38. Pop, E., Mann, D. A., Goodson, K. E. \& Dai, H. Electrical and thermal transport in metallic single-wall carbon nanotubes on insulating substrates. J. Appl. Phys. 101 (2007).

39. Mayhew, E. \& Prakash, V. Thermal conductivity of high performance carbon nanotube yarn-like fibers. J. Appl. Phys. 115, 174306 (2014).

40. Stauffer, D. \& Bunde, A. Introduction to Percolation Theory. Taylor Fr. London, https://doi.org/10.1063/1.2820231 (1994).

41. Nakajima, H. et al. Imaging of local structures affecting electrical transport properties of large graphene sheets by lock-in thermography. Sci. Adv. 5, eaau3407 (2019).

\section{Acknowledgements}

This paper is based on results obtained in a project commissioned by the New Energy and Industrial Technology Development Organization (NEDO).

\section{Author Contributions}

T.M. performed sample fabrication and measurements using the LIT method. S.A. and T.Y. fabricated composite materials. All authors discussed the results and wrote the manuscript.

\section{Additional Information}

Supplementary information accompanies this paper at https://doi.org/10.1038/s41598-019-50802-z.

Competing Interests: The authors declare no competing interests.

Publisher's note Springer Nature remains neutral with regard to jurisdictional claims in published maps and institutional affiliations.

(c) (i) Open Access This article is licensed under a Creative Commons Attribution 4.0 International

License, which permits use, sharing, adaptation, distribution and reproduction in any medium or format, as long as you give appropriate credit to the original author(s) and the source, provide a link to the Creative Commons license, and indicate if changes were made. The images or other third party material in this article are included in the article's Creative Commons license, unless indicated otherwise in a credit line to the material. If material is not included in the article's Creative Commons license and your intended use is not permitted by statutory regulation or exceeds the permitted use, you will need to obtain permission directly from the copyright holder. To view a copy of this license, visit http://creativecommons.org/licenses/by/4.0/.

(C) The Author(s) 2019 